\title{
Effect of Nanoparticles on the Performance of Solar Flat Plate Collectors
}

\author{
Mohammed Hamdan ${ }^{1 *}$, Maysa Sarsour ${ }^{1}$ \\ 1 Department of Mechanical Engineering, The University of Jordan, Amman, Jordan \\ * Corresponding author's e-mail: mhamdan@ju.edu.jo
}

\begin{abstract}
Solar energy constitutes superior renewable source due to its availability, natural distribution and no necessity for transportation. It can be designed to fulfill the requirements of power demands, and can work in synchronization with battery banks and diesel generators or any other power source to provide a continuous and stable power. Solar energy is already one of the best solutions to fulfill power demands. A solar thermal collector is a key element for the collection and conversion of solar energy into thermal energy. There are different types of solar collectors, the most important one is the conventional 'Flat-Plate' kind, which absorbs the incident radiation and converts it to thermal energy. The main objective of this work is to study the effect of Titanium Oxide $\left(\mathrm{TiO}_{2}\right)$ and Zinc Oxide $(\mathrm{ZnO})$ water-based nanofluids on the performance of solar flat plate collector. In order to achieve this objective, three identical flat plat solar collectors were used with appropriate instrumentations necessary to experimentally calculate their performance. The working fluid in the first collector was pure water, while in the second collector, it was Titanium Oxide water-based nanofluid and in the third one-Zinc Oxide water-based nanofluid. Two different values of volume fraction of both oxides were used as well. It was found that the addition of Titanium Oxide and Zinc Oxide nanoparticles leads to an increase in the efficiency of the solar collector. The efficiency of the collector was found to be maximum over all values of $\left(\mathrm{T}_{\text {in }}-\mathrm{T}_{\mathrm{amb}}\right)$ when a $0.2 \%$ volume fraction of Titanium Oxide was used with a maximum efficiency of $40 \%$, followed by the case of $0.6 \%$ volume fraction of Titanium Oxide. Furthermore, zinc oxide caused a maximum improvement in the performance of solar flat plate collector when $0.6 \%$ was used, followed by $0.2 \%$ concentration. Finally, it was found that titanium oxide is characterized by more pronounced improvement in the performance of solar flat plate collector.
\end{abstract}

Keywords: solar energy, nanoparticles, solar flat plate collector

\section{INTRODUCTION}

The whole world depends intensively on fossil fuels for producing its energy. They constitute non-renewable energy sources, so they are not available once they are fully consumed. They are also limited and depleted in a very fast way. Most of the fossil fuel resources and excessive consumption can cause serious environmental problems like air pollution, due to the release of carbon dioxide, sulphur oxide, and carbon monoxide.

Solar energy is a superior renewable source due to its availability, natural distribution and no necessity for transportation. It can be designed to fulfill the requirements of power demands, and can work in synchronization with battery banks and diesel generators or any other power source to provide a continuous and stable power. Solar energy is already one of the best solutions to fulfill power demands. A solar thermal collector is a key element for the collection and conversion of solar energy into thermal energy. There are different types of solar collectors, the most important one is the conventional 'Flat-Plate' kind, which absorbs the incident radiation and converts it to thermal energy.

Intensive work was conducted on domestic solar collectors. However, rather limited work was conducted on the improvement of collectors using nanotechnology. Kasaeian, et al. (2015), designed and manufactured a pilot of trough collector with a $0.7 \mathrm{~m}$ width and $2 \mathrm{~m}$ height reflector, which was 
made of a steel mirror. Then $0.2 \%$ and $0.3 \%$ carbon nanotube/oil based nanofluids were prepared as working fluid and tested in the pilot with the vacuumed copper absorber tube coated with black chrome. The results indicate that the global efficiency of the vacuumed tube is on averagely $11 \%$ higher than the bare tube efficiency. Shareef et al. (2015) showed that using $\mathrm{Al}_{2} \mathrm{O}_{3}$ has caused an $\mathrm{f}$ approximately four degrees rise in the outlet temperature compared to the di-ionized water while solar irradiance remains fairly unchanged. Said et al. (2014), analyzed theoretically the entropy generation, heat transfer improvement and pressure drop for a flat-plate solar collector operated with single wall carbon nanotubes (SWCNTs) based on nanofluids as an absorbing medium. Specific heat $(C p)$ of the nanofluid was measured using a PerkinElmer DSC 4000, and a density meter was used to measure the density of the nanofluid. Second law based on exergy analysis was carried out to evaluate the efficiency of the flat plate collector. It was noticed that the SWCNTs nanofluid minimized the entropy generation by $4.34 \%$ and improved the heat transfer coefficient by $15.33 \%$ theoretically, compared to water as an absorbing fluid. The pumping power penalty of nanofluid-operated solar collector was found to be $1.20 \%$ higher than in the case of water used as a working fluid.

Alim et al. (2013), analyzed theoretically the entropy generation, heat transfer enhancement capabilities and pressure drop of an absorbing medium with suspended nanoparticles $\left(\mathrm{Al}_{2} \mathrm{O}_{3}\right.$, $\mathrm{CuO}, \mathrm{SiO}_{2}, \mathrm{TiO}_{2}$ dispersed in water) inside a flat plate solar collector. It was found that the $\mathrm{CuO}$ nanofluid could reduce the entropy generation by $4.34 \%$ and enhance the heat transfer coefficient by $22.15 \%$, compared to water and additional pumping power of $1.58 \%$. Chaji et al. (2013), studied the effects of different nano particle concentrations of $\mathrm{TiO}_{2}$ in water as base fluid and the effect of increasing flow rates on the performance of a small flat plate solar collector (FPSC). It was found that both adding the $\mathrm{TiO}_{2}$ and increasing flow rates resulted in an improved overall efficiency of the collector. Gupta et al. (2013), investigated the effect of using $\mathrm{Al}_{2} \mathrm{O}_{3}$ nanofluid at variable flow rates. The experiment was conducted using distilled water and $0.005 \%$ volume fraction of $20 \mathrm{~nm}$ size $\mathrm{Al}_{2} \mathrm{O}_{3}$ nano-particles at flow rates of 1.5, 2 and $2.5^{2}$ liter per minute The authors concluded that the usage of nanofluid instead of water improved the optical and thermophysical characteristics of collector. The collector efficiency improvement by $8.1 \%$ and $4.2 \%$ has been noticed for 1.5 and 2 litter per minute flow rate of nanofluid, respectively. Optimum flow rate was found to be 2.5 and 2 litter per minute. Ladjevardi et al. (2013) have investigated the applications of nanofluids in direct absorptions of solar radiation in a solar collector. In this research, radiative transport equations, along with mass, momentum and energy equations, are solved together to simulate the operating characteristics of direct absorption solar collector. Different sizes and volume fractions of graphite nanoparticles were used to investigate the efficiency of solar receivers in absorption of solar energy, impacts on the harvested solar energy. The results of this study showed that by using graphite nanofluids having volume fraction around $0.025 \%$, more than $50 \%$ of incident irradiation energy was absorbed by just about $0.0045 \$ / \mathrm{L}$ increase in cost, while pure water solar collector can only absorb around $27 \%$ of the incident irradiation energy. Experiments conducted by Yousefi et al. (2012) showed that the usage of Triton X-100 surfactant in the noanofluid, in addition to $\mathrm{Al}_{2} \mathrm{O}_{3}$ of $0.2 \%$ volume fraction and particle size of $15 \mathrm{~nm}$, increased the efficiency by $28.3 \%$ in comparison to water. An additional study by Yousefi et al. (2012) confirmed the same finding related to the use of surfactant. Moreover, it showed that an increase in the concentration of multiwall carbon nanotube (MWCNT) from 0.2 to 0.4 weight fraction resulted in a substantial increase in the efficiency. Tyagi et al. (2009) investigated the feasibility of using a concentrating direct absorption solar collector (DAC) and compares its performance with that of a typical flat-plate collector with a nanofluid used as the absorbing medium. It was noticed that the presence of nanoparticles increases the absorption of incident radiation by more than nine times over that of pure water. It was found that under similar operating conditions, the efficiency of a direct absorption collector DAC using nanofluid as the working fluid was increased by $10 \%$ in relation to the one of a flat-plate collector. Generally, a DAC using nanofluids as the working fluid performs better than a flat-plate collector.

The main objective of this work is to study the effect pertaining to the addition of Titanium and Zinc Oxides nanoparticles to form water-based nanofluid as working fluid on the performance of the solar flat plate collector, using different values of concentrations of these oxides under Jordanian climate conditions. 


\section{EXPERIMENTAL PROCEDURE}

As a first step, three solar collectors were installed side by side on the metal frame that is facing south at the angle of 43 degrees. Then, the storage tank was installed in such a way that the hot fluid outlet from the collector is introduced directly into the tank. Stirrer is used to prevent sedimentation of solid nanoparticles and hence to maintain a homogenous mixture of nanofluid. The hot homogenous nanofluid is then introduced into a heat exchanger, where it is cooled down by the coolant water before it leaves the exchanger at relatively low temperature and is then introduced into the collector.

After smooth flow of fluid was achieved within the system, the flow rate of fluid was measured using a calibrated flow meter. With the flow rate kept fixed at $0.8 \mathrm{1} / \mathrm{min}$ and with $0.2 \%$ concentration of $\mathrm{TiO}_{2}$ and $\mathrm{ZnO}$ in the second and third collectors, the system is put into operation and the fluid is forced to flow through the collector using an electric pump. The amiant, together with the inlet and outlet fluids temperatures of each collector, were recorded on an hourly basis and stored within a Data Acquisition System (DAS). Finally, the incident solar radiation data was obtained directly from the weather station.

\section{RESULTS AND DISCUSSION}

Figure 2 represents the performance of the flat plate collector, which uses Titanium Oxide $\left(\mathrm{TiO}_{2}\right)$ nanofluid as a working fluid, with two different concentration values. The performance of the collector when pure water is used as a working fluid is also shown in the figure. As expected and as stated in theory, the general trend of the collector efficiency decreases linearly with the difference between inlet fluid and the ambient temperatures $\left(T_{\text {in }}-T_{\text {amb }}\right)$. As indicated in this figure, the collector exhibits the lowest performance when pure water is used a working fluid, with a maximum efficiency of $20 \%$ and this efficiency decreases linearly with the value of $\left(\mathrm{T}_{\mathrm{in}}-\mathrm{T}_{\mathrm{amb}}\right)$. On the other hand, the best performance of the collector was obtained when the volume concentration of nanoparticles is $0.2 \%$ with a maximum efficiency of the collector is slightly above $40 \%$ with a decreasing slope of 29.5. Furthermore, the performance of the collector is improved when a $\mathrm{TiO}_{2}$ concentration of $0.6 \%$. This increase in the efficiency of the collector is due to the fact that the thermal conductivity of the nanofluid is higher than that of pure water and hence more thermal energy is absorbed by the working fluid. It must be noted that the variation of thermal conductivity of the nanofluid with the volume concentration of nanoparticles is not exactly known. Consequently, and as indicated in literature, an optimum value of the nanoparticle concentration is usually to be found experimentally.

Figure 3 shows the efficiency of the flat plate collector with Zinc Oxide ( $\mathrm{ZnO})$ water-based nanofluid used as the working fluid. Two different concentration values of Zinc Oxide were used. The figure also shows the efficiency of the collector when pure water is used as a working fluid. As indicated the general trend, the efficiency of the collector decreases linearly with difference between inlet fluid and the ambient temperatures $\left(\mathrm{T}_{\text {in }}-\mathrm{T}_{\mathrm{amb}}\right)$. As it may be noticed in the figure, and in general, the performance of the collector is enhanced when Zinc Oxide is added to pure water to form the nanofluid. This enhancement

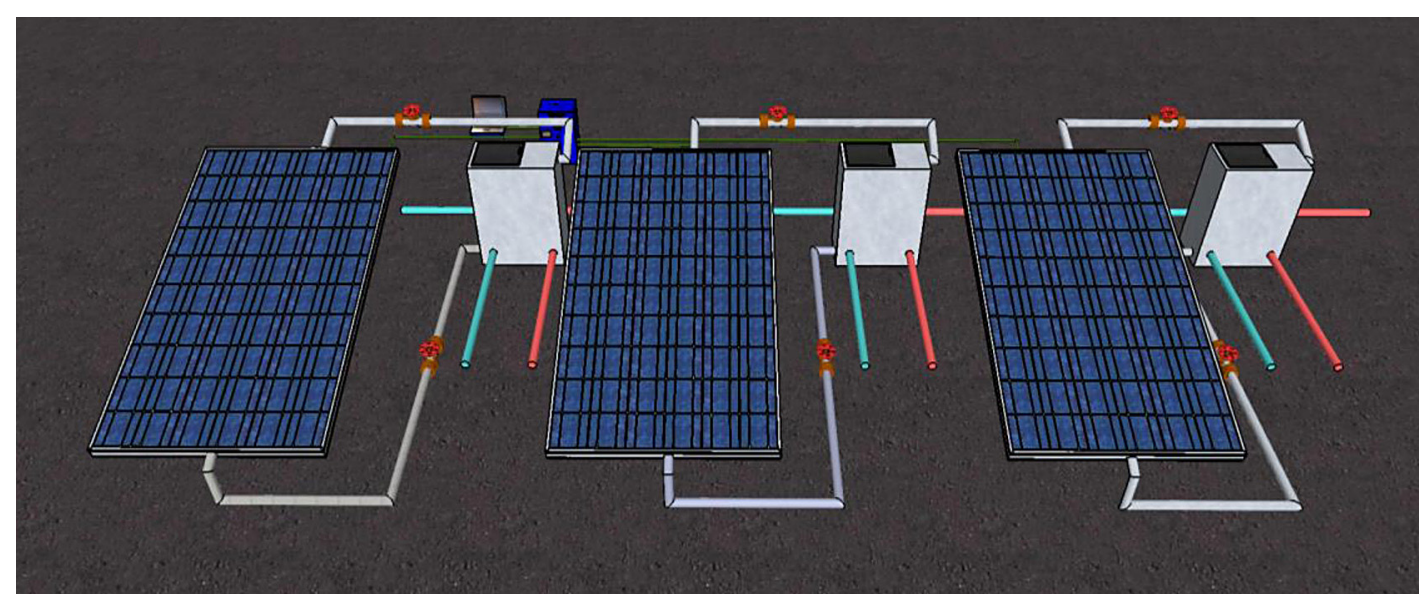

Figure 1. Experimental setup 


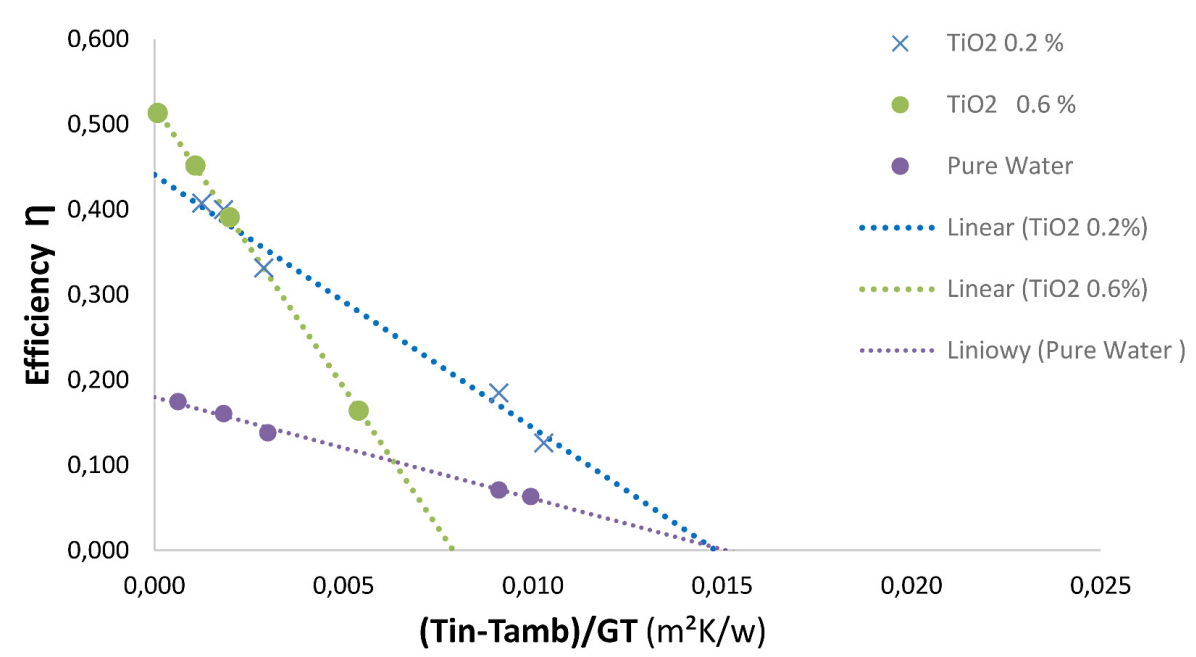

Figure 2. Efficiency of solar collector operated with $\mathrm{TiO}_{2}$ based nanofluids at different volume fraction

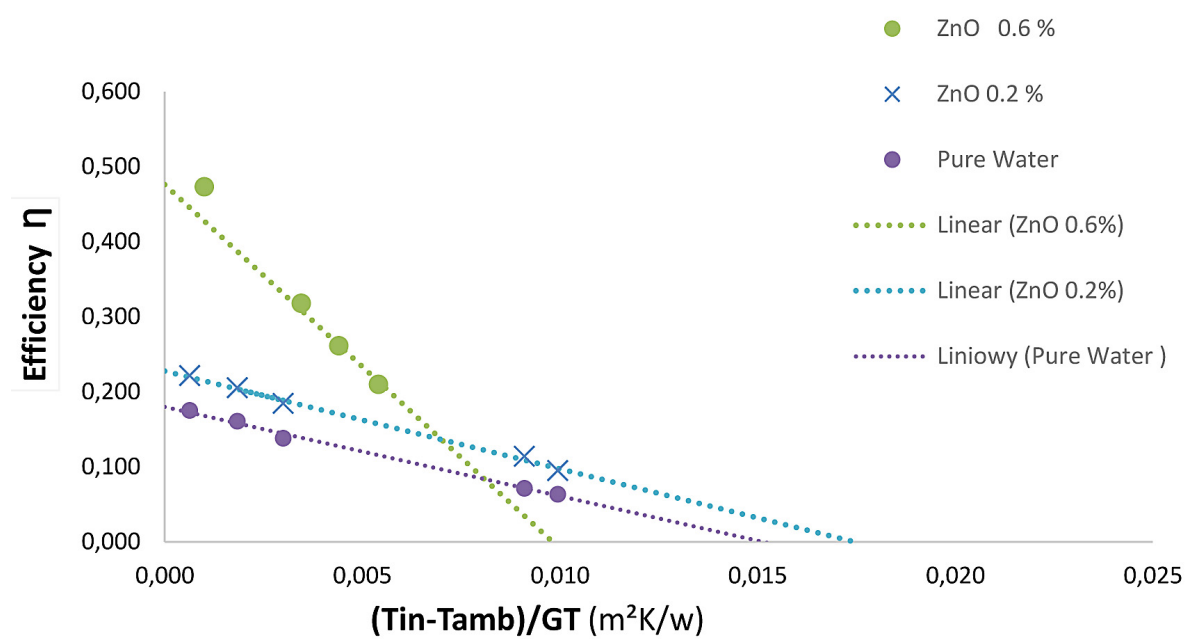

Figure 3. Efficiency of solar collector operated with $\mathrm{ZnO}$ based nanofluids at different volume fractions

in the performance is more pronounced at lower values of $\left(T_{\text {in }}-T_{\text {amb }}\right)$. It may be stated that under normal operating values of $\left(\mathrm{T}_{\text {in }}-\mathrm{T}_{\text {amb }}\right)$ and as shown in the figure, the increase in the efficiency of the collector is maximum when a concentration value of $\mathrm{ZnO}$ of $0.6 \%$ is used followed by a value of $0.2 \%$. As stated earlier, this variation in the efficiency is due to the increase in the thermal conductivity of the working nanofluid compared with that of pure water.

Figure 4 shows the effect of using $0.2 \%$ concentration of both Titanium and Zinc oxides. As indicated, the efficiency under almost over all range of values $\left(\mathrm{T}_{\mathrm{in}}-\mathrm{T}_{\text {amb }}\right)$ is maximum when Titanium Oxide is used, followed by the case when Zinc Oxide is added to water to form the working fluid. The efficiency reaches minimum values when pure water is used as working fluid. Under all conditions and in agreement with theory, the efficiency decreases linearly with $\left(\mathrm{T}_{\mathrm{in}}-\mathrm{T}_{\mathrm{amb}}\right)$. The rate, at which the efficiency decreases is more significant in the case of Titanium Oxide, while this rate is almost the same for the cases of both Zinc Oxide and pure water.

Figure 5 shows the effect of using $0.6 \%$ concentration of both Titanium and Zinc oxides. As indicated, the efficiency in almost the entire range of values $\left(\mathrm{T}_{\mathrm{in}}-\mathrm{T}_{\mathrm{amb}}\right)$ is maximum when Zinc oxide is used, followed by the case of Titanium Oxide. The efficiency reaches minimum values when pure water is used as working fluid with exception under very high values of $\left(\mathrm{T}_{\text {in }}-\mathrm{T}_{\text {amb }}\right)$.

Under all conditions and in agreement with theory, the efficiency decreases linearly with $\left(\mathrm{T}_{\text {in }}\right.$ $-\mathrm{T}_{\mathrm{amb}}$ ). The rate, at which the efficiency decreases is more significant in the case of Titanium Oxide followed by that of Zinc Oxide and it is the lowest when pure water is used as working fluid. 


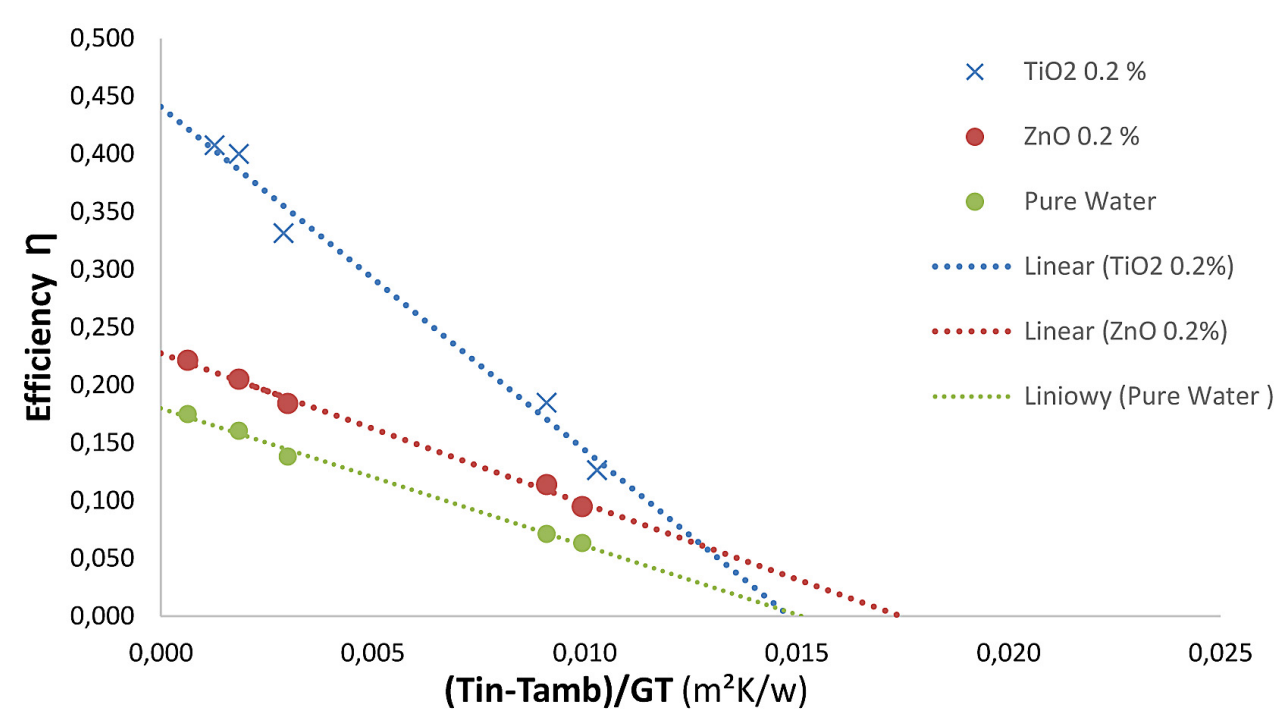

Figure 4. Efficiency of solar collector operated with $\mathrm{TiO}_{2}$ and $\mathrm{ZnO}$ based nanofluids at $0.2 \%$ volume fraction

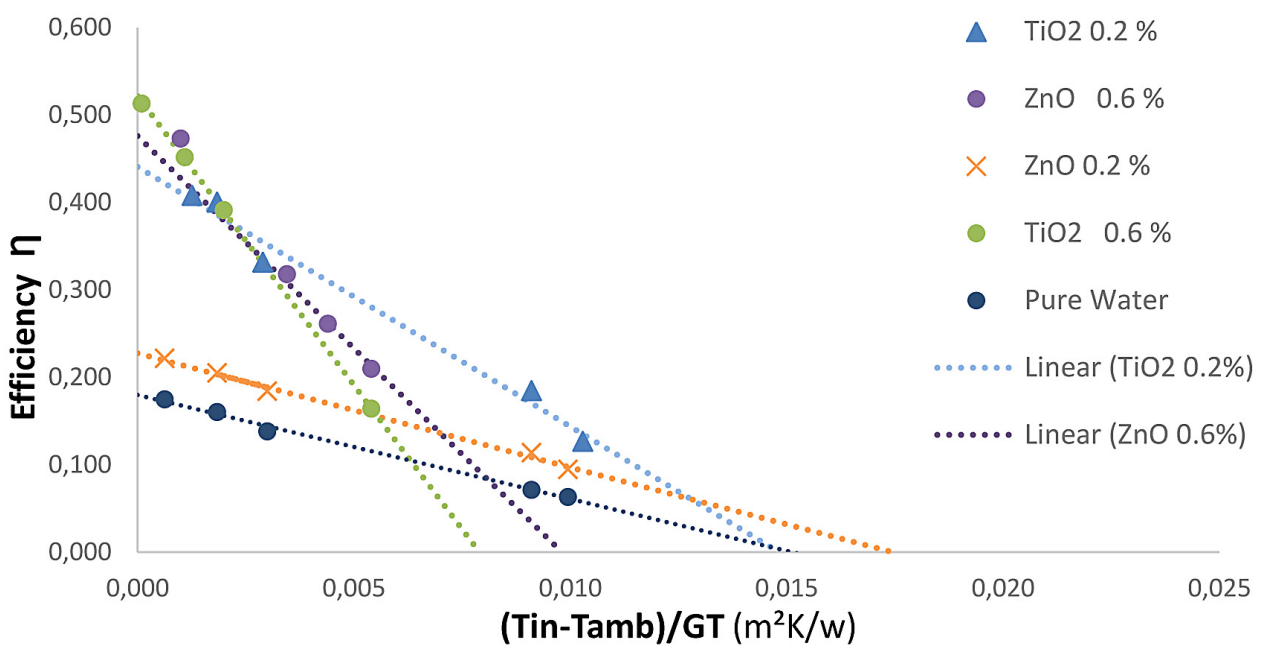

Figure 5. Efficiency of solar collector operated with $\mathrm{TiO}_{2}$ and $\mathrm{ZnO}$ based nanofluids at $0.6 \%$ volume fraction

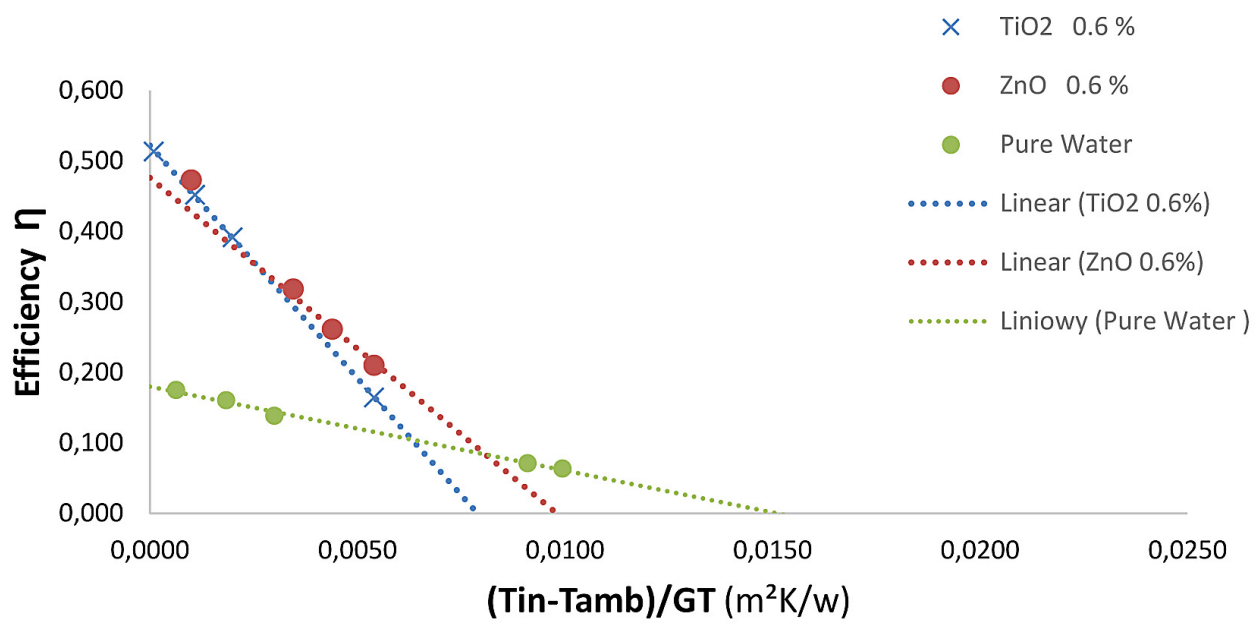

Figure 6. Efficiency of solar collector operated with $\mathrm{TiO}_{2}$ and $\mathrm{ZnO}$ based nanofluids at different values of volume fraction 
Table 1. The area under curves, efficiency equations, collector optical efficiency parameters and over all heat loss parameters

\begin{tabular}{|c|c|c|c|c|c|}
\hline Components & Volume fraction, $\%$ & Area under the curve $\times 100$ & $\eta$ equation & $\mathrm{FR}(\mathrm{T} \alpha)$ & FRUL \\
\hline $\mathrm{TiO} 2$ & 0.2 & 0.32 & $-29.456 x+.44$ & 0.44 & 29.45 \\
\hline TiO2 & 0.6 & 0.25 & $-70.38 x+0.52$ & 0.52 & 70.38 \\
\hline $\mathrm{ZnO}$ & 0.6 & 0.24 & $-48.557 x+0.48$ & 0.48 & 48.57 \\
\hline $\mathrm{ZnO}$ & 0.2 & 0.23 & $-13.302 x+0.23$ & 0.23 & 13.3 \\
\hline Pure water & & 0.18 & $-11.9 x+0.18$ & 0.18 & 11.9 \\
\hline
\end{tabular}

Figure 6 represents an overall summary of the present work, which shows the effect of adding the two types of nanoparticles, each with two different concentrations on the performance of the solar collector. As indicated in the figure and as stated earlier, the efficiency of the collector is maximum over all values of $\left(\mathrm{T}_{\text {in }}-\mathrm{T}_{\text {amb }}\right)$ when a $0.2 \%$ volume fraction of Titanium, followed by the case of $0.6 \%$ volume fraction of Titanium Oxide, then by the case when $0.6 \%$ volume fraction of Zinc Oxide was used. However, the lowest efficiency is achieved when pure water is used as a working fluid.

One way to evaluate the above-mentioned findings, is to estimate the areas under curve of each concentration shown in Sultan (2015). These areas were calculated and presented in Table 1. As indicated in the table, the maximum improvement in the collector performance corresponds to $0.2 \%$ concentration of Titanium Oxide with the area under curve of 0.32 . On the other hand, the least enhancement occurs of the performance was obtained when $0.2 \%$ of Zinc Oxide concentration was used.

\section{CONCLUSIONS}

In this experimental work, the effect of the addition of two types of nanoparticles (Titanium and Zinc Oxides) on the performance of a flat plat solar collector was investigated. From this work, the following may be concluded:

1. The addition of Titanium Oxide and Zinc Oxide in the nano scale leads to an improvement in the performance of the solar collector.

2. The efficiency of the collector is maximum over all values of $\left(\mathrm{T}_{\text {in }}-\mathrm{T}_{\text {amb }}\right)$ when a $0.2 \%$ volume fraction of Titanium Oxide is used with water as the working fluid, followed by the case of $0.6 \%$ volume fraction of Titanium Oxide, and then by the case when $0.6 \%$ volume fraction of Zinc Oxide was used.
3. The optimum volume fraction for $\mathrm{TiO}_{2}$ is $0.2 \%$; on the other hand, the optimum volume fraction for $\mathrm{ZnO}$ is $0.6 \%$.

4. The thermal efficiency of the solar collector reaches minimum values when pure water is used as a working fluid.

5. In general, the addition of Titanium Oxide nano particle to the working fluid has more pronounced effect on the efficiency than the addition of Zinc Oxide nanoparticles.

\section{REFERENCES}

1. Alim, M.A., Abdin, Z., Saidur, R., Hepbasli, A., Khairul, M.A. \& Rahim, N.A. 2013. Analyses of entropy generation and pressure drop for a conventional flat plate solar collector using different types of metal oxide nanofluids. Energy and Buildings, 66, 289-296.

2. Chaji, H., Ajabshirchi, Y., Esmaeilzadeh, E., Heris, S.Z., Hedayatizadeh, M. \& Kahani, M. 2013. Experimental study on thermal efficiency of flat plate solar collector using $\mathrm{TiO}_{2}$ water nanofluid. Modern Applied Science, 7(10), 60.

3. Gupta, H.K., Agrawal, G.D. \& Mathur, J. 2013. Investigations for effect of $\mathrm{Al}_{2} \mathrm{O}_{3}-\mathrm{H}_{2} \mathrm{O}$ nanofluid flow rate on the efficiency of direct absorption solar collector. Case Studies in Thermal Engineering, 5, 70-78.

4. Kasaeian, A., Daviran, S., Azarian, R.D. \& Rashidi, A. 2015. Performance evaluation and nanofluid using capability study of a solar parabolic trough collector. Energy Conversion and Management, 89, 368-375.

5. Ladjevardi, S.M., Asnaghi, A., Izadkhast, P.S. \& Kashani, A.H. 2013. Applicability of graphite nanofluids in direct solar energy absorption. Solar Energy, 94, 327-334.

6. Shareef, A.S., Abbod, M.H. \& Kadhim, S.Q. 2015. Experimental investigation on a flat plate solar collector using $\mathrm{Al}_{2} \mathrm{O}_{3}$ nanofluid as a heat transfer agent. International Journal of Energy and Environment, 6(4), 317. 
7. Sultan, K.F. 2015. Experimental evaluation of the thermal performance in the solar nanofluid heating system by using cupper and titanium oxide. The Iraqi Journal for Mechanical and Material Engineering, 15(4), 272.

8. Tyagi, H., Phelan, P. \& Prasher, R. 2009. Predicted efficiency of a low-temperature nanofluid-based direct absorption solar collector. Journal of Solar Energy engineering, 131(4), 041004.
9. Yousefi T., Veysi F., Shojaeizadeh E. \& Zinadini, S. 2012. An experimental investigation on the effect of $\mathrm{Al}_{2} \mathrm{O}_{3}-\mathrm{H}_{2} \mathrm{O}$ nanofluid on the efficiency of flat plate solar collectors. Renew Energy; 39(1), 293-298.

10. Yousefi, T., Shojaeizadeh, E., Veysi, F. \& Zinadini, S. 2012. An experimental investigation on the effect of $\mathrm{pH}$ variation of $\mathrm{MWCNT}-\mathrm{H}_{2} \mathrm{O}$ nanofluid on the efficiency of a flat-plate solar collector. Solar Energy, 86(2), 771-779. 